\title{
A hybrid double-dot in silicon
}

\author{
M. Fernando Gonzalez-Zalba用, Dominik Heiss $\{$ and Andrew J. \\ Ferguson \\ Microelectronics, Cavendish Laboratory, Cambridge University, Cambridge, UK \\ E-mail: ajf1006@cam.ac.uk
}

\begin{abstract}
We report electrical measurements of a single arsenic dopant atom in the tunnel-barrier of a silicon SET. As well as performing electrical characterization of the individual dopant, we study series electrical transport through the dopant and SET. We measure the triple points of this hybrid double dot, using simulations to support our results, and show that we can tune the electrostatic coupling between the two sub-systems.

PACS numbers: 85.30.Tv, 72.20.-i, 73.20.Hb, 73.23.Hk
\end{abstract}

$\ddagger$ Contributed equally to this work

$\S$ Contributed equally to this work 
The study of single dopants in silicon is motivated by the prospect of quantum computation with long-lived electronic and nuclear spins [1]. The observation of individual dopant states in nanoscale field effect transistors has been important progress towards this goal. Electrical transport spectroscopy has enabled positive identification of dopants [2, 3, 4] as well as investigations of their energy level structure in the presence of an interface [5, 6]. More recently, spin-read out of an dopant electronic state has been performed using a silicon single electron transistor (SET) to sense the occupancy of a nearby dopant [7]. The ability to measure the dopant spin state is important for future experiments that probe the electron and nuclear spin coherence of single dopants.

A parallel direction in silicon based quantum computation has been the development of few electron quantum dots, where the spin state of single confined electrons (or electron pairs) is of interest. This follows the progress made in the GaAs material system, but with the advantage of a reduced nuclear spin environment. Specifically, in double quantum dots the well-established mechanism of spin-blockade enables the singlet and triplet states to be distinguished [8, 9]. In GaAs, this has enabled experiments on gate defined few electron quantum dots allowing the investigation of spin lifetime, spin coherence and exchange interaction between electrons in the two dots [10, 11]. In silicon, spin blockade has been observed in double quantum dots [12, 13, 14], and spin measurements performed in single quantum dots [15, 16, 17].

In this article, we report the electrical characterization of double dot formed from a single arsenic atom and a silicon SET. This approach combines the research on dopants and quantum dots and could provide a new way to read out the long-lived spin state of a dopant using spin blockade. In contrast to an earlier study [18], our SET is gate defined allowing electrostatic control over both the dopant and SET, and consequent observation and analysis of the triple points.

The device fabrication starts by growth of a $10 \mathrm{~nm}$ sacrificial oxide on a highresistivity (>7000 $\Omega \mathrm{cm})$ (100) silicon wafer. Ohmic contacts are defined by optical lithography and ion implantation of phosphorus $\left(15 \mathrm{keV}, 10^{15} \mathrm{~cm}^{-2}\right)$ and dopants included by low-dose $\left(15 \mathrm{keV}, 10^{11} \mathrm{~cm}^{-2}\right)$ ion-implantation of arsenic. The sacrificial silicon oxide is removed after the implant and a $10 \mathrm{~nm} \mathrm{SiO}_{2}$ gate oxide is regrown at $850^{\circ} \mathrm{C}$, which also anneals out the implantation damage. We perform a forming gas anneal at $450^{\circ} \mathrm{C}$ for 30 min followed by a rapid thermal anneal for $15 \mathrm{~s}$ at $1050^{\circ} \mathrm{C}$ to reduce the interface trap and fixed oxide charge density. The As profile was calculated by an implantation Monte-Carlo simulator [19] and has a maximum at $10 \mathrm{~nm}$ from the interface and a density of $4 \times 10^{16} \mathrm{~cm}^{-3}$. By comparison, the residual phosphorous doping is estimated to be smaller than $10^{12} \mathrm{~cm}^{-2}$. We note that a larger As density at the interface is expected due to segregation to the interface during the thermal processing [20].

Subsequent to the silicon processing, surface gates are fabricated by electron beam lithography and thermal evaporation of aluminium. Figure 1 shows a scanning electron microscopy image of an identical device and its schematic cross-section. In a first step, two gates $40 \mathrm{~nm}$ wide and $100 \mathrm{~nm}$ apart are defined by evaporation of a $25 \mathrm{~nm}$ thick layer 
of aluminium. They are used to form the tuneable source and drain tunnel barriers. After thermal oxidation at $150^{\circ} \mathrm{C}$ for 5 min creating $5 \mathrm{~nm}$ aluminium oxide, a second electrically-independent $60 \mathrm{~nm}$ thick top-gate is deposited over the barriers. This topgate defines the $120 \mathrm{~nm}$ wide channel of the SET and leads that overlap with the doped ohmic contacts. Prior to measurement the samples undergo a nitrogen ambient post-fabrication anneal at $350^{\circ} \mathrm{C}$ for $15 \mathrm{~min}$. The interface trap density, measured on simultaneously processed field effect transistors, by means of low frequency split $\mathrm{C}-\mathrm{V}$ method [21], is $1.8 \times 10^{10} \mathrm{~cm}^{-2}$.

The device can be operated in one of the three different modes depicted in figure 1. SET (c), single dopant (d), and hybrid dopant-SET device (e). For SET operation, the top-gate is set well above threshold and the tunnel barriers are biased to locally deplete the electron accumulation layer underneath. This forms an isolated island of electrons between the barriers leading to Coulomb oscillations in charge transport [22]. The second mode allows transport spectroscopy of individual As impurity atoms in the silicon substrate. The top-gate and drain tunnel barrier are biased well above threshold allowing the study of subthreshold phenomena underneath the source tunnel barrier. Finally, the third mode of operation permits the formation of a tuneable capacitively coupled dopant-SET hybrid. Here, the SET is defined while one of the barriers is tuned in resonance with a dopant transition.

Electrical transport measurements are performed at the base temperature of a dilution refrigerator (electron temperature of $200 \mathrm{mK}$ ) using radio-frequency reflectometry [23]. This technique probes the reflection coefficient of a resonant circuit that includes the device as a circuit element. As the impedance, in our case the differential conductance, of the device changes so does the reflection coefficient of the resonant circuit. This technique allows an increase of bandwidth over a standard dc or lock-in measurement. The sample was embedded in a rf-tank circuit formed by a surface mount $390 \mathrm{nH}$ inductor and a parasitic capacitance $(500 \mathrm{fF})$ to ground. An rf-carrier signal is applied to the source of the device at the resonant frequency of $360 \mathrm{MHz}$ and the cryo-amplified reflected signal is homodyne detected [24]. A bias tee on the sample board permits the simultaneous measurement of the two terminal dc conductance.

To form the SET tunnel barriers, a bias of $560 \mathrm{mV}$ and $314 \mathrm{mV}$ is applied to the source and drain barriers respectively. Periodic Coulomb diamonds are observed over a large range of top-gate bias (figure 2), similar to earlier studies on undoped devices 22. From the diamonds we extract a voltage period of $\Delta \mathrm{V}_{t g}=4.8 \mathrm{mV}$ and a charging energy of $\mathrm{E}_{c}^{S E T}=1.4 \pm 0.1 \mathrm{meV}$, which corresponds to $\alpha_{S E T}=C_{t g} / C_{S E T}=0.29$, where $C_{t g}$ is the capacitive coupling of the topgate to the SET and $C_{S E T}$ is the total SET capacitance. Due to our gate geometry, where the top-gate controls electron density in the island as well as the leads, we are not able to deplete the SET to the few electron limit. However, few electron quantum dots have been measured in a similar geometry but with separated gates controlling the leads and the island [25, 26, 27] .

We now describe electrical transport in the sub-threshold region beneath a single barrier, where we focus on the source barrier. The top-gate and drain barrier are set 
above threshold $\left(\mathrm{V}_{t g}=1.94 \mathrm{~V}\right)$, while the rf-response is measured as a function of $\mathrm{V}_{b s}$ (figure $3(\mathrm{a})$ ) Below the conduction band edge $\left(\mathrm{V}_{b s}=430 \mathrm{mV}\right)$, the data show electrical transport through states labeled 1, 2, 3 in figure 3. These features appear at the same bias voltages in several cool down cycles. We identify these states as dopants in the barrier due to their charging energies, as extracted from (figure 3 (c,d)), being larger than $10 \mathrm{meV}$. In contrast, when we measure undoped samples, parasitic quantum dots are formed in the channel due to disorder at the interface, and these have a charging energy below $5 \mathrm{meV}$ [28]. To further investigate the nature of these states, we measured the line shape of the tunnel current as a function of temperature (symbols in figure $3(b)$ ). This is fitted to the expected behaviour for resonant tunnelling through a discrete state (lines in figure 3(b)). In particular the maximum current increases with decreasing temperature, in contrast to Coulomb blockade through a multi-level system such as the SET. This is consistent with transport though a dopant with well separated energy levels $\left(\Delta E>k_{B} T\right)$. Therefore, we attribute the transitions labelled 1, 2 and 3 to Arsenic dopants, which typically show charging energies on the order of 29-35 meV close to the $\mathrm{Si} / \mathrm{SiO}_{2}$ interface [2, 5].

We next examine the coupling, given by $\alpha=\mathrm{C}_{b s} / \mathrm{C}_{\Sigma}$, of the barrier gate to the different dopant transitions. This coupling is a direct translation of $\mathrm{V}_{b s}$ to energy change on the dopant site. The obtained values were $\alpha_{1}=0.21, \alpha_{2}=0.10, \alpha_{3}=0.08$. We expect the electrostatic characteristics of the device to change when changing the biasing conditions from $\mathrm{V}_{b s}=430 \mathrm{mV}$ to $-630 \mathrm{mV}$. The capacitive coupling of a dopant to the gate electrode $\left(C_{b s}\right)$ and the contacts $\left(\mathrm{C}_{s}, \mathrm{C}_{d}\right)$ is inherently changed due to screening effects and changes of the effective barrier width as a function of $\mathrm{V}_{b s}$. As a result, it is not possible to identify which transitions stem from the same dopant site by comparing these values alone.

We perform magneto-spectroscopy on these states, applying an in-plane magnetic field perpendicular to the current direction (figure 3 (e-g)). The peak position is converted to a chemical potential shift using $\alpha$, and is in agreement with the expected Zeeman shift of $58 \mu \mathrm{eV} / \mathrm{T}$ for an electron with g-factor $=2$. Transitions 1 and 3 shift to lower energies corresponding to transport through the lower Zeeman sub-level. This behaviour is expected for tunnelling through an ionized donor $\left(\mathrm{D}^{+}-\mathrm{D}^{0}\right.$ transition) [2, 5]. In contrast, transition 2 shifts to higher energies, suggesting the lower Zeeman level is already occupied and tunnelling takes place through an already occupied (neutral) donor $\left(\mathrm{D}^{0}-\mathrm{D}^{-}\right.$transition). Accordingly, we identify the three sub-threshold peaks as follows: 1 corresponds to $\mathrm{D}^{+}-\mathrm{D}^{0}$ transition of an As donor; 2 to the corresponding $\mathrm{D}^{0}$ $\mathrm{D}^{-}$transition; and 3 is identified as the $\mathrm{D}^{+}-\mathrm{D}^{0}$ transition of an additional As donor. For typical donor charging energies of $29-35 \mathrm{meV}$ we expect the corresponding $\mathrm{D}^{0}-\mathrm{D}^{-}$ transition to be around $\mathrm{V}_{b s}=400 \mathrm{mV}$, where several conductance peaks are observed that cannot be clearly identified.

Following this, the charging energy for transition 1 and 2 is determined as $E_{c}=73 \mathrm{meV}$. Here, we use a average value of $\alpha_{1,2}=0.155$ and bias voltage difference $\Delta \mathrm{V}_{b s}=470 \mathrm{mV}$. Such an increased charging energy has not been observed so far, but 
may be a consequence our relatively large dopant density, the presence of another ionized As atom leading to an increased ionization energy [29, 30]. Another possibility is that, an orbital Stark shift [31] increases the observed charging energy. The electric field at the dopant site varies for the two transitions, due to the difference in biasing conditions. Additionally, a weaker screening effect induced by the metallic dominated interface is expected for the charged impurity atom in comparison to the neutral dopant [32].

Investigation of the drain barrier revealed two additional resonant tunnelling features consistent with transport through a neutral donor. As in the case of transition 3 the corresponding $\mathrm{D}^{0}-\mathrm{D}^{-}$transition could not be clearly identified. Consequently, these states were not investigated further.

We now turn our attention to the hybrid dopant-SET system, investigating the sequential transport through a single dopant and the SET. A positive voltage of $\mathrm{V}_{t g}=2 \mathrm{~V}$ is applied to the top-gate while the source barrier gate voltage is set to $\mathrm{V}_{b d}=375 \mathrm{mV}$. The drain barrier is tuned such that transport is governed by tunnelling through transition 2 , since in this configuration the strongest dopant-SET is observed.

Again using rf-reflectometry, we measured the hybrid system formed by transition 2 and the SET, as a function of top-gate voltage $\mathrm{V}_{t g}$ and barrier $\mathrm{V}_{b s}$ (figure 4(a)). As in a double quantum dot, electrical conduction occurs at points with three-fold degeneracy of the charge state, known as triple points [33. Transport through the system can be described by a capacitance model, and we simulated the differential conductance to compare to the rf-reflectometry measurement (figure 4(b)). The software SIMON [34] was used to calculate tunnelling probabilities by Monte-Carlo simulation. The SET was described as a metallic island with a constant density of states. The dopant was simulated as a discrete energy level system represented by delta functions. An excitation voltage of $125 \mu \mathrm{V}$, corresponding to $-97 \mathrm{dBm}$ of rf-power at the tank circuit, was used to obtain the differential conductance.

For increasing top-gate voltage we observe the charge transitions of the SET with a gate period of $\Delta \mathrm{V}_{t g}=4.8 \mathrm{mV}$ in agreement with the characterization of the SET alone (figure 4(b)). The dopant transition has been identified as $\mathrm{D}^{0}-\mathrm{D}^{-}$, therefore, the electron number increases from 1 to 2 for increasing barrier voltage $\mathrm{V}_{b s}$. Weak lines of increased conductance are observable at the charge transitions due to elastic cotunnelling processes [35, 36].

In the finite bias regime $\left|V_{s d}\right|>0$ the conductance regions change from triplepoints to triangles (figure 5(a)). We measure (figure 5(b,c)) and simulate (figure 5(d,e)) these bias-triangles for both bias polarities. The dimensions of the bias triangles are related to the bias voltage through the corresponding values of $\alpha, \alpha_{b s}=e\left|\mathrm{~V}_{s d}\right| / \delta \mathrm{V}_{b s}$, $\alpha_{t g}=e\left|\mathrm{~V}_{s d}\right| / \delta \mathrm{V}_{t g}$. From the measurement presented in figure 5(b,c), we can extract $\alpha_{b s}=0.16$ and $\alpha_{t g}=0.28$ for the dopant site and the SET, respectively. Despite the change in biasing, this is in reasonable agreement with the values obtained from isolated measurement of the dopant $\left(\alpha_{2}=0.10\right)$ and the $\operatorname{SET}\left(\alpha_{S E T}=0.29\right)$ stability diagram.

An additional feature parallel to the dopant line is visible within the bias triangle (figure 5(b)), at an energy of $0.27 \pm 0.03 \mathrm{meV}$ from the ground state. Such lines of 
increased differential conductance within the bias triangle can arise from modulation of density of states in the leads [37]. Alternatively, such features can be observed when the energy level of an excited dopant state enters the bias window. We cannot distinguish the origin of this line, but note that in the 2e configuration of a dopant a valley-spin excited state around $1 \mathrm{meV}$ has been observed [38. We do not detect the additional line for $\mathrm{V}_{s d}=0.8 \mathrm{meV}$, which is a consequence of the asymmetry in the hybrid system.

We will now discuss the coupling of the dopant and the SET. A measure for the electrostatic coupling of the SET to the dopant is the ratio $C_{m} / C_{S E T}=\Delta \mathrm{V}_{b s}^{m} / \Delta \mathrm{V}_{b s}$ [39], where $C_{m}$ is the mutual capacitance between SET and dopant. The induced voltage change of the dopant line at the triple points $\Delta \mathrm{V}_{b s}^{m}$ can be extracted from the measurement, as indicated in figure 6(d) and $\Delta V_{b s}=470 \mathrm{mV}$ is the separation of the $\mathrm{D}^{+}$ $\mathrm{D}^{0}$ and the $\mathrm{D}^{0}-\mathrm{D}^{-}$transition of the dopant. The data in figure 4 (a) shows $\Delta \mathrm{V}_{b s}^{m}=2.3 \mathrm{mV}$, resulting in $C_{m} / C_{S E T}=0.008$. In analogy the electrostatic effect of the dopant on

the SET is $C_{m} / C_{A s}=\Delta \mathrm{V}_{t g}^{m} / \Delta \mathrm{V}_{t g}=0.53$. Using the previously determined total capacitance of the dopant $\mathrm{C}_{A s}=2.1 \mathrm{aF}$ and the $\mathrm{SET} \mathrm{C}_{S E T}=116 \mathrm{aF}$ a mutual capacity of $C_{m}=1.0 \pm 0.2 \mathrm{aF}$ is estimated. Under these conditions the maximum source-drain current measured at the triple point for $\mathrm{V}_{s d}=-0.65 \mathrm{mV}$ is $130 \mathrm{pA}$, which corresponds to a tunnelling time of 1.2 ns between dopant and SET.

Furthermore, the dopant-SET coupling can be tuned by changing the bias on the drain tunnel barrier $V_{b d}$ (figure 6(a-c)). In figure 6(e,f) the electrostatic coupling $C_{m} / C_{S E T}$ and $C_{m} / C_{A s}$ are plotted as a function of $V_{b d}$. As the voltage on the drain tunnel barrier is increased, the separation of the triple points decreases, which corresponds to a reduced electrostatic coupling between the dopant and the SET. This change arises partly from a reduced capacitive coupling $C_{m}$ as the SET island extends further away from the dopant site, partly from an increased $C_{S E T}$ as the SET is coupled more strongly to the drain lead.

To conclude, we have studied a hybrid double-dot formed by coupling an dopant and an SET in series. In transport spectroscopy, we observe triple points and bias triangles characteristic of a double quantum dot. The analogy with the double quantum dot could be taken further by reducing the SET to the few electron limit, which will allow spin-blockade to be observed. This will provide a new tool for the investigation of single electron spins on dopants in silicon.

\section{Acknowledgments}

We are grateful for support of EPSRC grant EP/H016872/1 and the Hitachi Cambridge Laboratory. AJF was supported by the Hitachi Research Fellowship. We acknowledge discussions with David Williams. MFGZ was supported by Obra Social La Caixa fellowship. 


\section{References}

[1] B. E. Kane. A silicon-based nuclear spin quantum computer. Nature, 393(6681):133-137, May 1998.

[2] H. Sellier, G. P. Lansbergen, J. Caro, S. Rogge, N. Collaert, I. Ferain, M. Jurczak, and S. Biesemans. Transport spectroscopy of a single dopant in a gated silicon nanowire. Physical Review Letters, 97(20):206805, 2006.

[3] Y. Ono, K. Nishiguchi, A. Fujiwara, H. Yamaguchi, H. Inokawa, and Y. Takahashi. Conductance modulation by individual acceptors in si nanoscale field-effect transistors. Applied Physics Letters, 90(10):102106, 2007.

[4] Kuan Yen Tan, Kok Wai Chan, Mikko Mottonen, Andrea Morello, Changyi Yang, Jessica van Donkelaar, Andrew Alves, Juha-Matti Pirkkalainen, David N. Jamieson, Robert G. Clark, and Andrew S. Dzurak. Transport spectroscopy of single phosphorus donors in a silicon nanoscale transistor. Nano Letters, 10(1):11-15, 2010. PMID: 19950969.

[5] G. P. Lansbergen, R. Rahman, C. J. Wellard, I. Woo, J. Caro, N. Collaert, S. Biesemans, G. Klimeck, L. C. L. Hollenberg, and S. Rogge. Gate-induced quantum-confinement transition of a single dopant atom in a silicon finfet. Nature Physics, 4(8):656-661, 2008.

[6] M. Pierre, R. Wacquez, X. Jehl, M. Sanquer, M. Vinet, and O. Cueto. Single-donor ionization energies in a nanoscale cmos channel. Nature Nanotechnology, 5(2):133-137, February 2010.

[7] Andrea Morello, Jarryd J. Pla, Floris A. Zwanenburg, Kok W. Chan, Kuan Y. Tan, Hans Huebl, Mikko Mottonen, Christopher D. Nugroho, Changyi Yang, Jessica A. van Donkelaar, Andrew D. C. Alves, David N. Jamieson, Christopher C. Escott, Lloyd C. L. Hollenberg, Robert G. Clark, and Andrew S. Dzurak. Single-shot readout of an electron spin in silicon. Nature, 467(7316):687-691, October 2010.

[8] K. Ono, D. G. Austing, Y. Tokura, and S. Tarucha. Current rectification by pauli exclusion in a weakly coupled double quantum dot system. Science, 297(5585):1313-1317, 2002.

[9] A. C. Johnson, J. R. Petta, C. M. Marcus, M. P. Hanson, and A. C. Gossard. Singlet-triplet spin blockade and charge sensing in a few-electron double quantum dot. Physical Review B, $72: 165308$, Oct 2005.

[10] R. Hanson, L. P. Kouwenhoven, J. R. Petta, S. Tarucha, and L. M. K. Vandersypen. Spins in few-electron quantum dots. Reviews of Modern Physics, 79(4):1217-1265, 2007.

[11] J. R. Petta, A. C. Johnson, J. M. Taylor, E. A. Laird, A. Yacoby, M. D. Lukin, C. M. Marcus, M. P. Hanson, and A. C. Gossard. Coherent manipulation of coupled electron spins in semiconductor quantum dots. Science, 309(5744):2180-2184, 2005.

[12] Nakul Shaji, C. B. Simmons, Madhu Thalakulam, Levente J. Klein, Hua Qin, H. Luo, D. E. Savage, M. G. Lagally, A. J. Rimberg, R. Joynt, M. Friesen, R. H. Blick, S. N. Coppersmith, and M. A. Eriksson. Spin blockade and lifetime-enhanced transport in a few-electron si/sige double quantum dot. Nature Physics, 4(7):540-544, July 2008.

[13] H. W. Liu, T. Fujisawa, Y. Ono, H. Inokawa, A. Fujiwara, K. Takashina, and Y. Hirayama. Paulispin-blockade transport through a silicon double quantum dot. Physical Review B, 77:073310, Feb 2008.

[14] M. G. Borselli, K. Eng, E. T. Croke, B. M. Maune, B. Huang, R. S. Ross, A. A. Kiselev, P. W. Deelman, I. Alvarado-Rodriguez, A. E. Schmitz, M. Sokolich, K. S. Holabird, T. M. Hazard, M. F. Gyure, and A. T. Hunter. Pauli spin blockade in undoped si/sige two-electron double quantum dots. Applied Physics Letters, 99(6):063109, 2011.

[15] M. Xiao, M. G. House, and H. W. Jiang. Measurement of the spin relaxation time of single electrons in a silicon metal-oxide-semiconductor-based quantum dot. Physical Review Letters, 104:096801, Mar 2010.

[16] Robert R. Hayes, Andrey A. Kiselev, Matthew G. Borselli, Steven S. Bui, Edward T. Croke III, Peter W. Deelman, Brett M. Maune, Ivan Milosavljevic, Jeong-Sun Moon, Richard S. Ross, Adele E. Schmitz, Mark F. , Gyure, and Andrew T. Hunter. Lifetime measurements (t1) of 
electron spins in si/sige quantum dots. arXiv, 0908:0173, 2009.

[17] C. B. Simmons, J. R. Prance, B. J. Van Bael, Teck Seng Koh, Zhan Shi, D. E. Savage, M. G. Lagally, R. Joynt, Mark Friesen, S. N. Coppersmith, and M. A. Eriksson. Tunable spin loading and $T_{1}$ of a silicon spin qubit measured by single-shot readout. Physical Review Letters, 106:156804, Apr 2011.

[18] V. N. Golovach, X. Jehl, M. Houzet, M. Pierre, B. Roche, M. Sanquer, and L. I. Glazman. Singledopant resonance in a single-electron transistor. Physical Review B, 83(7):075401, Feb 2011.

[20] A.S Grove, Leistiko J.O., and C.T. Sah. Redistribution of acceptor and donor impurities during thermal oxidation of silicon. Journal of Applied Physics, 35(9):2695-2701, 1964.

[21] J. Kommen. Investigation of most channel conductance in weak inversion. Solid State Electronics, 16(7):801-810, 1973.

[22] S. J. Angus, A. J. Ferguson, A. S. Dzurak, and R. G. Clark. Gate-defined quantum dots in intrinsic silicon. Nano Letters, 7(7):2051-2055, July 2007.

[23] R. J. Schoelkopf, P. Wahlgren, A. A. Kozhevnikov, P. Delsing, and D. E. Prober. The radiofrequency single-electron transistor (rf-set): A fast and ultrasensitive electrometer. Science, 280(5367):1238-1242, 1998.

[24] S. J. Angus, A. J. Ferguson, A. S. Dzurak, and R. G. Clark. A silicon radio-frequency single electron transistor. Applied Physics Letters, 92(11):112103, 2008.

[25] W. H. Lim, F. A. Zwanenburg, H. Huebl, M. Mttnen, K. W. Chan, A. Morello, and A. S. Dzurak. Observation of the single-electron regime in a highly tunable silicon quantum dot. Applied Physics Letters, 95(24):242102, 2009.

[26] M. Xiao, M. G. House, and H. W. Jiang. Parallel spin filling and energy spectroscopy in fewelectron si metal-on-semiconductor-based quantum dots. Applied Physics Letters, 97(3):032103, 2010 .

[27] W H Lim, C H Yang, F A Zwanenburg, and A S Dzurak. Spin filling of valley orbit states in a silicon quantum dot. Nanotechnology, 22(33):335704, 2011.

[28] G. J. Podd, S. J. Angus, D. A. Williams, and A. J. Ferguson. Charge sensing in intrinsic silicon quantum dots. Applied Physics Letters, 96(8), 2010.

[29] B. Koiller, X. D. Hu, and S. Das Sarma. Electric-field driven donor-based charge qubits in semiconductors. Physical Review B, 73(4), 2006.

[30] Rajib Rahman, Richard P. Muller, James E. Levy, Malcolm S. Carroll, Gerhard Klimeck, Andrew D. Greentree, and Lloyd C. L. Hollenberg. Coherent electron transport by adiabatic passage in an imperfect donor chain. Physical Review B, 82(15):155315, Oct 2010.

[31] R. Rahman, G. P. Lansbergen, S. H. Park, J. Verduijn, G. Klimeck, S. Rogge, and L. C. L. Hollenberg. Orbital stark effect and quantum confinement transition of donors in silicon. Physical Review B, 80(16), 2009.

[32] R. Rahman, G. P. Lansbergen, J. Verduijn, G. C. Tettamanzi, S. H. Park, N. Collaert, S. Biesemans, G. Klimeck, L. C. L. Hollenberg, and S. Rogge. Electric field reduced charging energies and two-electron bound excited states of single donors in silicon. Phys. Rev. B, 84:115428, Sep 2011.

[33] W. G. van der Wiel, S. De Franceschi, J. M. Elzerman, T. Fujisawa, S. Tarucha, and L. P. Kouwenhoven. Electron transport through double quantum dots. Reviews of Modern Physics, 75(1):1-22, Dec 2002.

$[34]$

[35] S. De Franceschi, S. Sasaki, J. M. Elzerman, W. G. van der Wiel, S. Tarucha, and L. P. Kouwenhoven. Electron cotunneling in a semiconductor quantum dot. Physical Review Letters, 86(5):878-881, 2001.

[36] H. W. Liu, T. Fujisawa, T. Hayashi, and Y. Hirayama. Pauli spin blockade in cotunneling transport through a double quantum dot. Physical Review B, 72(16):161305, Oct 2005.

[37] Martin Fuechsle, MahapatraS., ZwanenburgF. A., Mark Friesen, ErikssonM. A., and Michelle Y. 
Simmons. Spectroscopy of few-electron single-crystal silicon quantum dots. Nature Nanotechnology, 5(7):502-505, July 2010.

[38] R. Lansbergen, G.P.and Rahman, J. Verduijn, G.C. Tettamanzi, N. Collaert, S. Biesemans, G. Klimeck, L.C.L. Hollenberg, and S. Rogge. Lifetime enhanced transport in silicon due to spin and valley blockade. arXiv, 1008:1381, 2011.

[39] Gento Yamahata, Yoshishige Tsuchiya, Shunri Oda, Zahid A. K. Durrani, and Hiroshi Mizuta. Control of electrostatic coupling observed for silicon double quantum dot structures. Japanese Journal of Applied Physics, 47(6):4820-4826, 2008. 


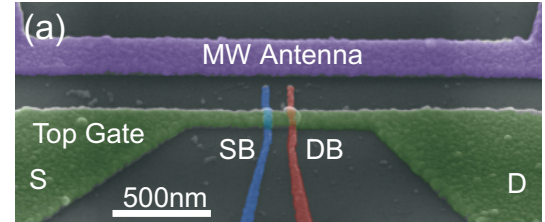

(b)

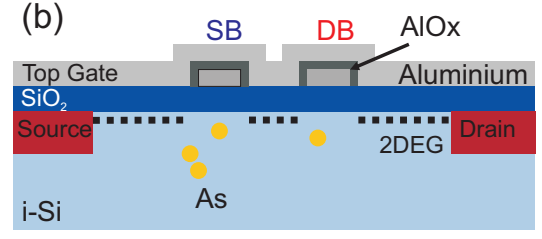

(c) SB TG DB

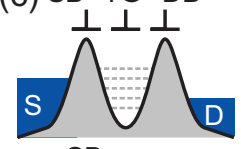

(d) $\stackrel{\mathrm{SB}}{\perp}$

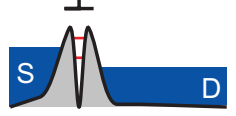

(e) SB TG DB

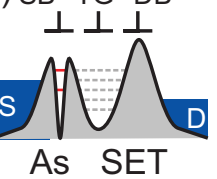

Figure 1. (a) Scanning electron microscopy image of an identical device. An on-chip coplanar stripline, for electron spin resonance, is included next to the sample but is not used in this experiment. (b) Schematic representation of the sample cross-section. (c-e) Schematic representation of the modes of device operation: (c) single electron transistor,(d) single dopant device, (e) dopant-SET hybrid device.

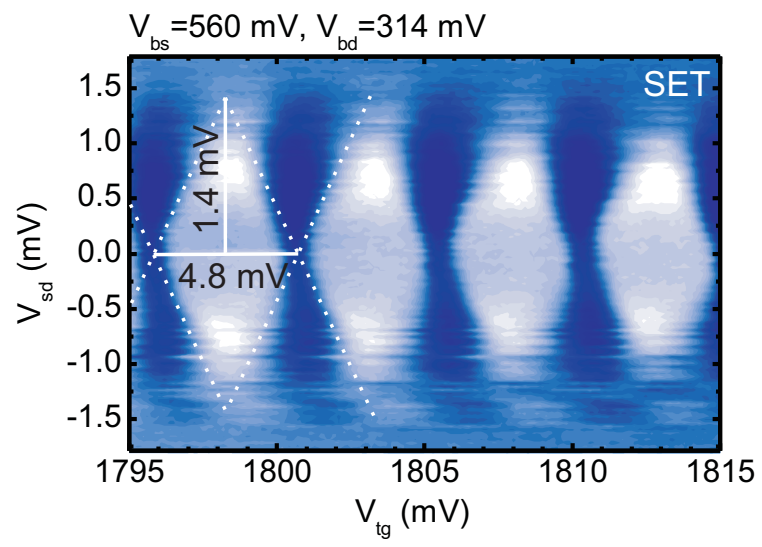

Figure 2. Measurement of the device in SET operation mode using rf-reflectometry. Coulomb diamonds with a gate period of $4.8 \mathrm{mV}$ and a charging energy of $1.4 \mathrm{meV}$ are observed. 

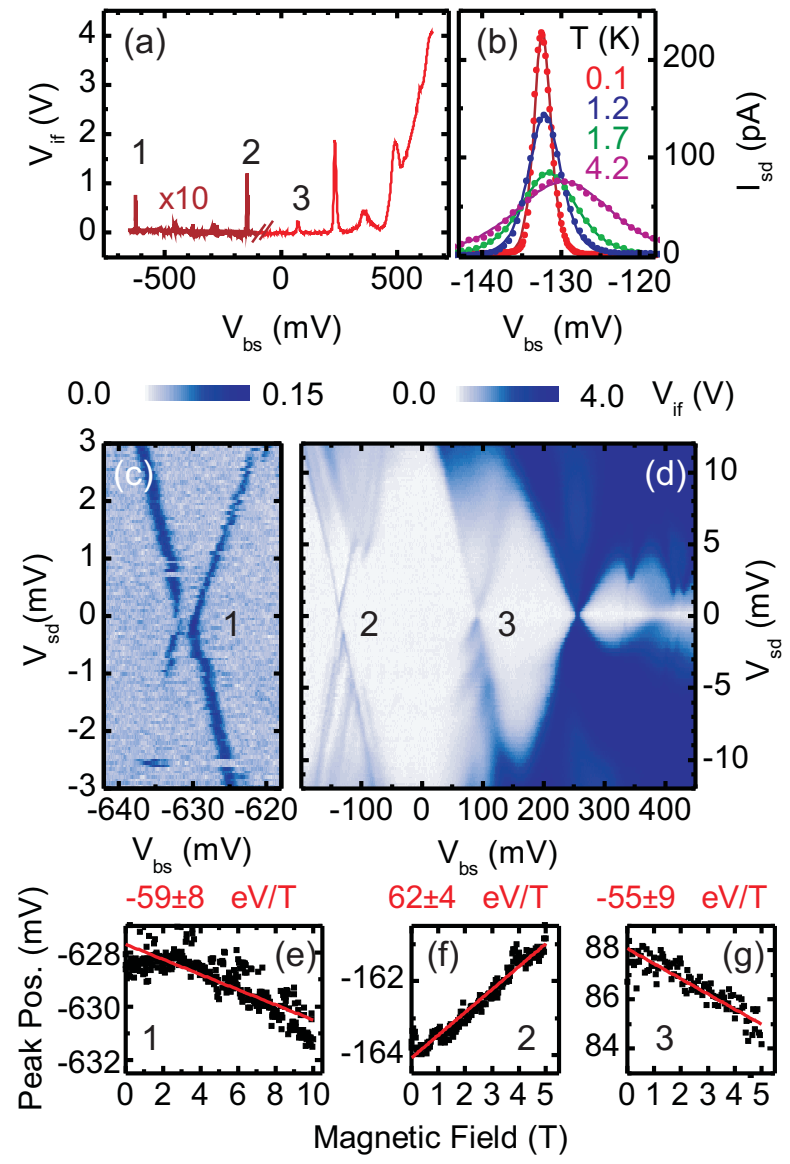

Figure 3. (a) Turn-off characteristics of the drain tunnelling barrier. Subthreshhold features labelled 1,2 and 3 are observed. (b) Tunnelling current of transition 2 as a function of temperature. In this measurement $\mathrm{V}_{s d}=-0.1 \mathrm{mV}$. $(\mathrm{c}, \mathrm{d})$ Coulomb diamonds of the subthreshold features. (e-g) Magnetic field dependence of the peak position of transition 1,2 and 3 , respectively. 

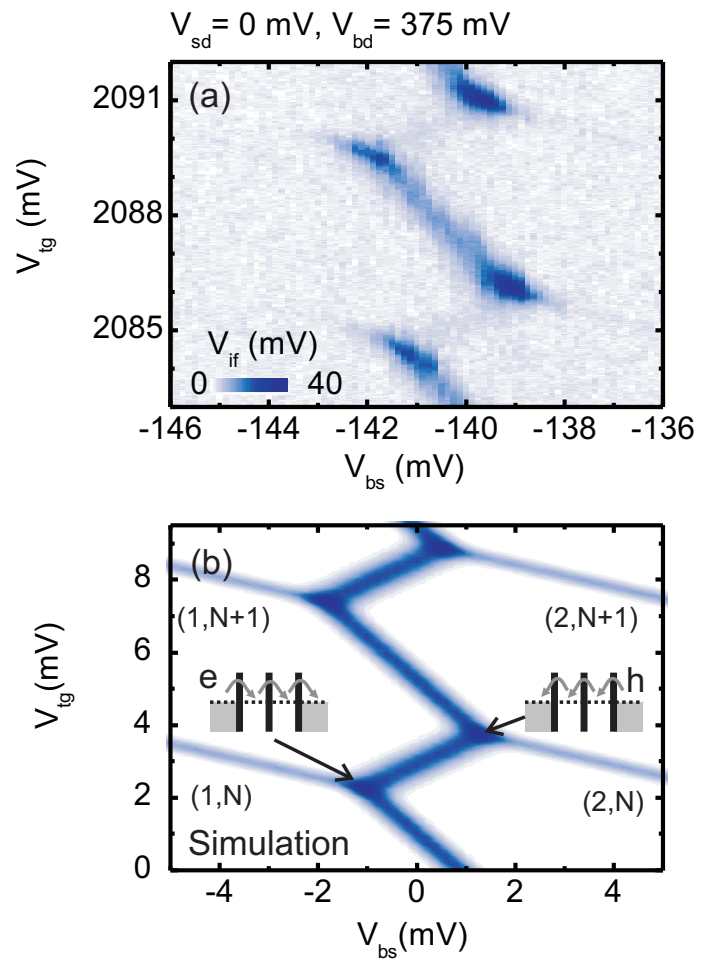

Figure 4. (a) Rf-reflectometry measurement as a function of top-gate bias $\mathrm{V}_{t g}$ and source barrier bias $\mathrm{V}_{b s}$. Four triple points can be observed within this range. (b) Simulation of the measurement presented in (a). The charge state is labelled in the form $(n, m)$, where $n$ denotes the number of electrons on the dopant site, while $\mathrm{m}$ stands for the electron number on the SET. The inset illustrates the difference between electron and hole transport process. 
(a)
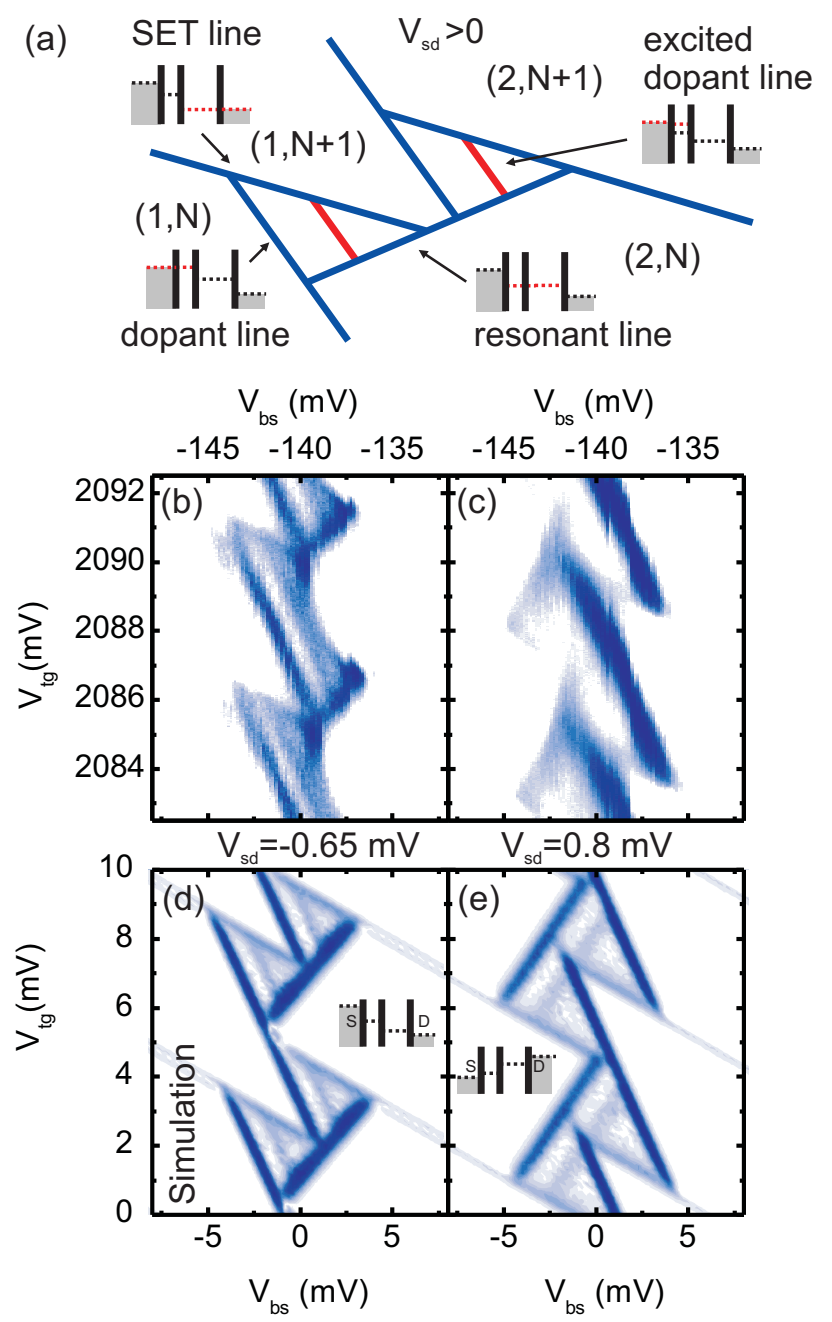

Figure 5. (a) Schematic representation of the bias triangles observed for $\mathrm{V}_{s d}>0$. The resonant conditions defining the borders of the triangles are indicated. $(\mathrm{b}, \mathrm{c})$ Rf-reflectometry measurement of the hybrid system for $\mathrm{V}_{s d}=-0.65$ and $0.8 \mathrm{mV}$, respectively. (d,e) Simulation of the measurement presented in $(b, c)$. The SET is described by the capacitive coupling to drain $\mathrm{C}_{d}=74 \mathrm{aF}$, the top-gate $\mathrm{C}_{t g}=34 \mathrm{aF}$ and a total capacitance $\mathrm{C}_{S E T}=116 \mathrm{aF}$. For the dopant we find the source $\left(\mathrm{C}_{s}=0.7 \mathrm{aF}\right)$, barrier $\left(\mathrm{C}_{b s}=0.34\right)$ and total $\left(\mathrm{C}_{A s}=2.1 \mathrm{aF}\right)$ capacitance. 

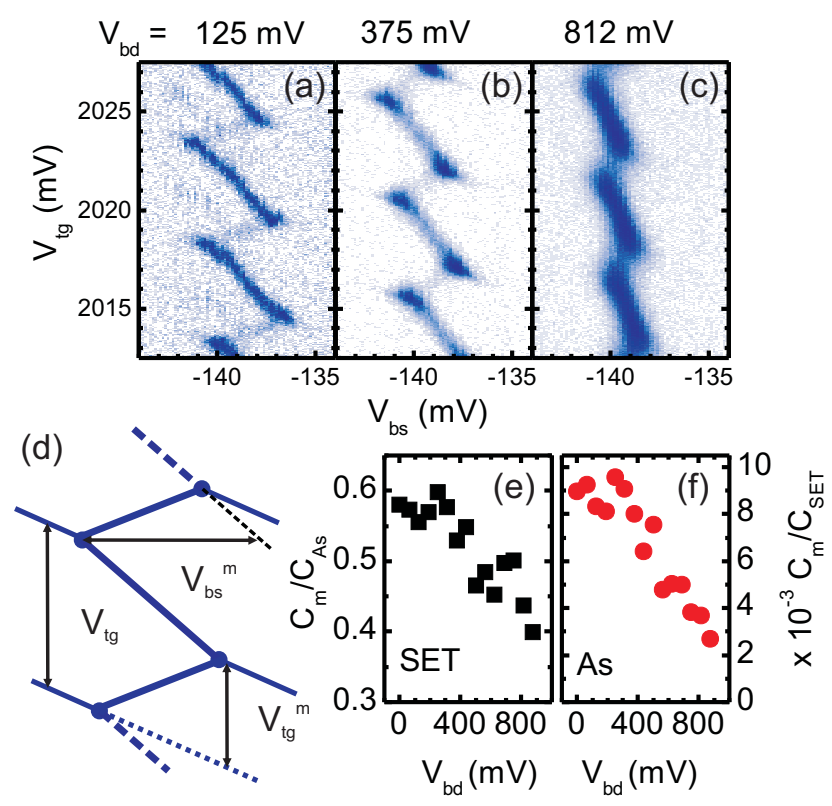

Figure 6. (a-c) The triple points of the hybrid system for drain barrier voltages $\mathrm{V}_{b d}=125,375,812 \mathrm{mV}$, respectively. (d) Schematic representation of the triple point measurement indicating the voltage separation $\Delta \mathrm{V}_{b s}^{m}, \Delta \mathrm{V}_{t g}$ and $\Delta \mathrm{V}_{t g}^{m}$. (e,f) Electrostatic coupling $C_{m} / C_{A s}$ and $C_{m} / C_{S E T}$ as a function of drain barrier bias $\mathrm{V}_{b d}$. 